Ann. Biol. anim. Bioch. Biophys., I974, 14 (4-B), 82I-827.

\title{
VARIATIONS NYGTHÉMERALES DE LA MAGNÉSÉMIE CHEZ LE RAT : INFLUENCE DU JEÛNE ET DE LA RÉPLÉTION STOMACALE
}

\author{
Y. RAYSSIGUIER et P. LARVOR \\ avec la collaboration technique de M. Genest \\ Station de Physiopathologie de la Nutrition, \\ Centre de Recherches zootechniques et vétérinaires, I. N.R. A., \\ Theix, 63110 Beaumont
}

\section{RÉSUMÉ}

Il existe chez le Rat des variations cycliques de la magnésémie différentes de celles observées pour le calcium et le phosphore sanguin.

Ces variations ne sont pas sous le contrôle de la médullo-surrénale mais ne semblent pas résulter uniquement de l'absorption intestinale de magnésium pendant la période nocturne de prise de nourriture. La mise à jeun modifie la réponse hypermagnésémiante de la médullo-surrénale à certains traitements. Le jeûne entraîne une élévation de la magnésémie supprimée par une réplétion stomacale non nutritive chez l'animal normal, mais pas chez l'animal déficient en $\mathrm{Mg}$.

\section{INTRODUC'TION}

Des variations nycthémérales de la magnésurie ont été décrites mais l'influence đu régime alimentaire, déterminante dans le taux d'élimination urinaire du magnésium, a souvent été oubliée et les observations chez 1'Homme ne sont pas toujours concordantes (DOE, VENNES et FLINK, I960; RIZzo, I965; BRISCOE et RAGAN, I966) et les rythmes d'excrétion urinaire ont des caractéristiques très variables d'un sujet à l'autre (DARLU et HENROTTE, I972). Aux larges variations de la magnésurie s'opposerait la stabilité remarquable de la magnésémie qui ne serait influencée ni par le jeûne, ni par la prise de nourriture (STutzzman et Amatuzio, I952 ; Heaton et HodGKInson, I963 ; Drenick, Hunt et Swendseid, I969). Chez 1'Homme, il n'a pas été mis en évidence de modifications nycthémérales du magnésium sanguin (revue bibliographique : HENROTTE et DURLACH, I97I) mais d'une façon générale les résultats demandent à être précisés 
La possibilité de disposer de lots homogènes de rats Sherman, élevés au laboratoire dans les mêmes conditions (nourriture, éclairage, température), nous a conduit à déterminer le taux de magnésium plasmatique au cours du nycthémère et à étudier l'influence du jeûne, de la prise de nourriture et l'interaction avec différents traitements.

\section{MATÉRIEL ET MÉTHODES}

\section{Animaux}

Des rats Sherman mâles pesant de 150 à $200 \mathrm{~g}$, sont adaptés à des périodes alternatives de lumière artificielle $(6 \mathrm{~h}$ à $\mathbf{I} 9 \mathrm{~h}$ ) et d'obscurité ( $\mathrm{I} 9 \mathrm{~h}$ à $6 \mathrm{~h}$ ) et répartis au hasard dans les groupes nécessaires aux essais. Ils ont à leur disposition une alimentation standard du commerce (croquettes Sanders).

\section{Traitements divers}

Les animaux carencés sont nourris pendant 8 jours avec un régime semi-synthétique carencé en magnésium (RAYSSIGUIER, LARVOR et BARLET, I973). Les rats privés de médullo-surrénale sont opérés selon la technique de INGLE et GRIFFITH (1963) et sont utilisés une semaine après l'opération. La réplétion non nutritive de l'estomac est obtenue par sondage gastrique d'une préparation barytée (BARYXR Laboratoires Seclo) deux heures avant la prise de sang. La pilocarpine est injectée à la dose de $200 \mathrm{mg} / \mathrm{kg}$ par voie intrapéritonéale et le sang recueilli une heure après.

\section{Prélèvements sanguins et analyses}

Le sang est prélevé par ponction cardiaque sous anesthésie à l'éther. Dans le plasma hépariné, on dose le magnésium et le calcium par absorption atomique sclon les méthodes décrites par Perkin-Elmer Corp. (I97I) et le phosphore par colorimétrie (Kalckar, I947).

\section{Analyses statistiques}

Les moyennes sont comparées selon le test $t$ de Student ou par analyse de variance.

\section{RÉSULTATS}

\section{I. - Variations nycthémérales de la magnésémie, de la calcémie et de la phosphatémie}

Les rats en alimentation normale sont sacrifiés toutes les 3 heures. La figure I montre les variations de la magnésémie, de la calcémie et de la phosphatémie au cours d'une période de 24 heures. Le taux du magnésium plasmatique atteint un maximum pendant la période obscure. L'élévation de la magnésémie est suivie d'une baisse très rapide. Le calcium plasmatique présente un maximum pendant la première partie de la période claire et un minimum au début de la période obscure. Le phosphore plasmatique atteint un minimum au milieu de la période obscure et un maximum au début de la période claire. Les différences entre les valeurs maximales et minimales sont hautement significatives $(\mathrm{P}<\mathrm{O}, \mathrm{OI})$ pour $\mathrm{Mg}$, Ca et $\mathrm{P}$. L'ablation des médullosurrénales ne modifie pas les variations de la magnésémie au cours du nycthémère (tabl. I). 


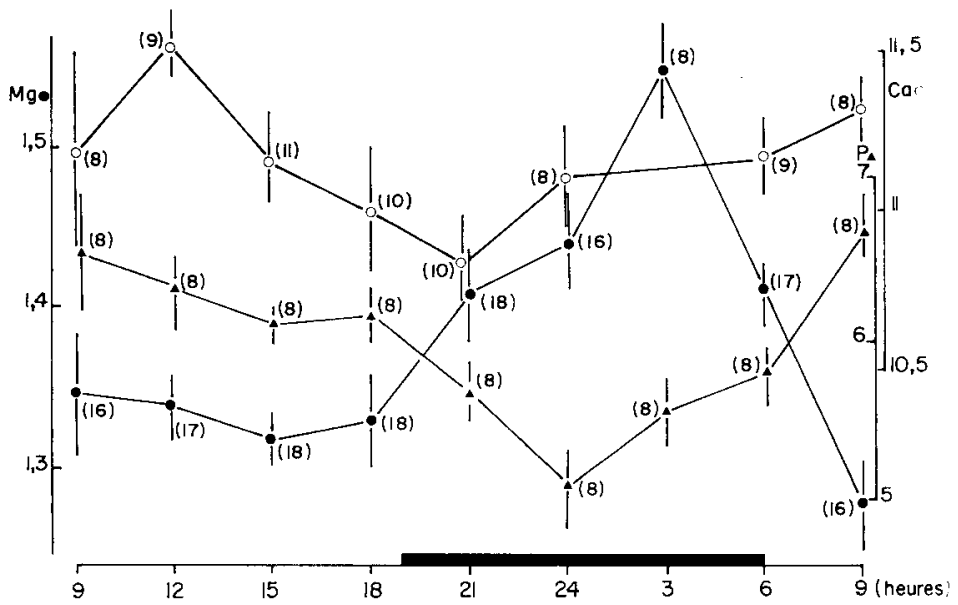

Fig. I. - Variations nycthémérales de la magnésémie (•), de la calcémie (o) et de la phosphatémie (4) en $\mathrm{mg} / \mathrm{I}$ oo $\mathrm{ml}$ chez le Rat

Moyenne \pm erreur type ; (nombre d'animaux) (I9-6 h = période obscure)

FIG. I. - Cyclic variations of plasma magnesium (•), calcium (O) and phosphate (4) level in Rat (mg/roo $\mathrm{ml}$ )

Mean \pm S. E. (number of animals) $(\mathrm{r} 9-6 \mathrm{~h}=$ dark period)

\section{TABLEAU I}

Influence du jếne et de l'ablation des médullo-surrénales (MX) sur les variations de la magnésémie (résultats exprimés en p. roo de la valeur à la fin de la période claire) Moyenne \pm erreur type (nombre de rats).

Effect of fasting and adrenal demedullation (MX) on cyclic variations of magnesemra. (Results expressed as p. Ioo of level at the end of the period of light) Mean $\pm S$. E. (number of rats).

\begin{tabular}{|c|c|c|c|}
\hline & $3 \mathrm{~h}$ & $9 \mathrm{~h}$ & $\begin{array}{l}\text { Signification } \\
\text { Significance } \\
\quad 3 \mathrm{~h} / 9 \mathrm{~h}\end{array}$ \\
\hline $\begin{array}{c}\text { Témoins (alimentes) } \\
\text { Controls }\end{array}$ & $\begin{array}{c}16,1 \pm 2,3 \\
(8)\end{array}$ & $\begin{array}{c}-1,6 \pm 3,0 \\
(8)\end{array}$ & $\mathrm{P}<0,01$ \\
\hline $\begin{array}{l}\text { A jeun } \\
\text { Unfed }\end{array}$ & $+\underset{(8)}{14,1 \pm 2,5}$ & $+\underset{(8)}{10,3} \pm 1,8$ & NS \\
\hline MX & $+\underset{(5)}{11,1 \pm 2,3}$ & $0,3 \pm 1,9$ & $P<0,01$ \\
\hline \multicolumn{4}{|l|}{$\begin{array}{l}\text { Signification } \\
\text { Significance }\end{array}$} \\
\hline $\begin{array}{l}\text { A jeun/témoins } \\
\text { Unfed/controls }\end{array}$ & NS & $P<0,01$ & \\
\hline $\begin{array}{l}\mathrm{MX} / \text { témoins } \\
M X / \text { controls }\end{array}$ & NS & NS & \\
\hline
\end{tabular}

Annales de Biologie animale. - I974. 


\section{TABLEAU 2}

Effet du jeûne de courte durée sur la magnésémie du Rat en régime normal ou carencé en magnésium et infuence de la réplétion de l'estomac (magnésémie en $\mathrm{mg} / \mathrm{I} 00 \mathrm{ml}$, moyenne \pm erreur type (nombre d'animaux). Signification de la différence entre animaux à jeun/alimentés $\mathrm{P}<0, \mathrm{OI}$ ).

Effect of short time fasting on magnesemia in Rat with $M g$ supplemented or $M g$ deficient diet and effect of gastric distension. (Magnesemia $\mathrm{mg} / \mathrm{l}$ oo $\mathrm{ml}$, mean $\pm \mathrm{S}$. E. (number of animals). Significance : Unfed/fed $\mathrm{P}<0,0 \mathrm{I})$.

\begin{tabular}{c|c|c|c}
\hline $\begin{array}{c}\text { Régime } \\
\text { Diet }\end{array}$ & $\begin{array}{c}\text { Alimentés } \\
\text { Fed }\end{array}$ & $\begin{array}{c}\text { A jeun } \\
\text { Unfed }\end{array}$ & $\begin{array}{c}\text { Réplétion } \\
\text { Gastric distension }\end{array}$ \\
\hline $\begin{array}{c}\text { Normal } \\
M \text { supplemented }\end{array}$ & $\begin{array}{c}1,39 \pm 0,02 \\
(16)\end{array}$ & $\begin{array}{c}1,55 \pm 0,02 \\
(15)\end{array}$ & $1,40 \frac{1}{(15)} 0,03$ \\
\hline $\begin{array}{c}\text { Carencé } \\
M g \text { deficient }\end{array}$ & $0,23 \frac{ \pm}{(7)} 0,02$ & $0,74 \frac{ \pm 0,05}{(7)} 0$ & $0,75 \pm 0,06$ \\
\hline \hline
\end{tabular}

\section{TABLEAU 3}

Effet de la pilocarpine (200 $\mathrm{mg} / \mathrm{kg}$ par voie intrapéritonéale) chez le Rat à jeun ou alimenté

(même expression des résultats que pour le tableau 2)

Effect of intraperioneal pilocarpine injection in fed or unfed rat (same expression of results than for table 2 )

\begin{tabular}{|c|c|c|}
\hline & 0 & Pilocarpine \\
\hline $\begin{array}{c}\text { A jeun } \\
\text { Unfed }\end{array}$ & 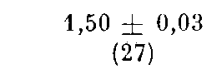 & $1,62 \underset{(27)}{ \pm} 0,03$ \\
\hline $\begin{array}{l}\text { Alimenté } \\
\text { Fed }\end{array}$ & $1,34 \underset{(27)}{ \pm} 0,02$ & $1,68{\underset{(27)}{ \pm}}^{ \pm} 0,04$ \\
\hline Analyse de variance & \multirow{2}{*}{\multicolumn{2}{|c|}{$\begin{array}{l}\text { Effet pilocarpine } \mathrm{P}<0,005 \\
\text { Interaction jê̂ne/pilocarpine } \mathrm{P}<0,005 \\
\text { Pilocarpine effect } P<0,005 \\
\text { Unfed/pilocarpine interaction } P<0,005\end{array}$}} \\
\hline Analysis of variance & & \\
\hline
\end{tabular}




\section{2. - Effet du jeûne et de la réplétion de l'estomac}

La mise à jeun des animatix pendant la période obscure (on sait que, chez le Rat, cette période correspond à la période active de prise de nourriture) n'empêche par l'élévation de la magnésémie pendant la première phase de la période obscure mais supprime la baisse très rapide de la magnésémie observée chez les rats alimentés (tabl. I). I a mise à jeun de brève durée ( 16 h à $24 \mathrm{~h}$ ) se traduit par une élévation significative de la magnésémie chez le Rat, en alimentation normale ou carencée en magnésium. Chez le Rat à jeun, la réplétion de l'estomac supprime l'hypermagnésémie mais seulement si l'animal n'est pas en déficit magnésique (tabl. 2).

\section{3. - Effet du jê̂ne sur la réponse à certains traitements}

I,a réponse hypermagnésémiante à la pilocarpine (tabl. 3) est diminuée chez l'animal à jeun $(\mathrm{P}<0,005)$.

\section{DISCUSSION}

Les variations nycthémérales de la magnésémie sont assez différentes de celles de la calcémie et de la phosphatémie déjà observées par Milnaud, Perault-Staub et STAUb (I972).

L'élévation de la magnésémie pendant la première phase de la période obscure coïncide avec l'augmentation de l'absorption intestinale de magnésium mais il faut remarquer l'augmentation comparable de la magnésémie des animaux à jeun pendant une période de temps similaire. Che' les rats alimentés, la baisse de la magnésémie dans la deuxième phase de la période obscure est à rapprocher de l'effet de la réplétion stomacale susceptible de diminuer la magnésémie élevée des animaux à jeun.

Il est à remarquer les conséquences différentes de la mise à jeun des différentes espèces : (les durées de privation de nourriture n'étant pas toujours comparables) pas de changement de la magnésémie chez 1'Homme, hypomagnésémie chez les ruminants (AlLCROFT et BURNS, I968), hypermagnésémie chez le Rat. BoIs et JASMIN (I97I) avaient souligné que l'état du jeûne relatif améliorait la magnésémie et diminuait les manifestations érythémateuses du déficit magnésique chez le Rat. Nos résultats montrent 1'élévation brutale de la magnésémie chez le Rat déficient en magnésium après seulement une nuit de jeûne et le peu de valeur qu'il convient d'attacher au taux du magnésium plasmatique pour apprécier un déficit magnésique (la magnésémie đes animaux à jeun carencés demeure cependant inférieure à celle des témoins à jeun). Diverses hypothèses peuvent être envisagées pour expliquer l'effet du jeûne et de la réplétion de l'estomac en particulier, une fuite urinaire de magnésium pourrait intervenir dans la baisse de la magnésémie après charge stomacale (Hodgkinson et HEaton, I965) et le catabolisme par libération de magnésium tissulaire pourrait contribuer à l'augmentation de la magnésémie lors du jeûne mais une participation endocrinienne ne peut être exclue a priori pour expliquer ces divers phénomènes. La médullo-surrénale ne semble pas influencer les variations journa- 
lières de la magnésémie mais la mise à jeun modifie la réponse hypermagnésémiante après injection de pilocarpine, réaction qui se produit par l'intermédiaire de la sécrétion de la médullo-surrénale (RAYSSIGUIER et LARVOR, I972). L'intervention d'autres glandes endocrines comme les parathyroïdes et la thyroïde (LARvor et DURI,ACH, I97I) serait à rechercher.

En conclusion, ces résultats montrent les variations importantes de la magnésémie au cours du nycthémère ainsi que l'influence du jeûne et de la réplétion stomacale. D'autres recherches seront nécessaires pour établir le déterminisme endocrinien des phénomènes observés.

Reçu pour publication en avril 1974.

\title{
SUMMARY
}

\author{
DIURNAL VARIATIONS OF PLASMA MAGNESIUM LEVEI, \\ IN THE RAT : EFFECT OF FASTING AND GASTRIC DISTENSION
}

In rats, the plasma magnesium level shows diurnal cycles (fig. I). These variations are not only related to magnesium intake. During the night hypermagnesemia is observed, and plasma magnesium concentration falls when there is magnesium absorption. Pilocarpine injection induces hypermagnesemia by adrenal medullary hypersecretion (RAYSSIGUIER and LARVOR, 1972). A decrease in hypermagnesemic response to injected pilocarpine is recorded in unfed rats, but the medulla is not involved in the diurnal cycles of magnesium level (tables $\mathbf{I}$ and 3 ).

When rats are fasted for a short time, the plasma magnesium concentration increases in $\mathrm{Mg}$ hormal or deficient rats and decreases after gastric distension in $\mathrm{Mg}$ normal rats (table 2). Other numoral factors, such as the thyroid and parathyroid, may be involved.

\section{RÉFÉRENCES BIBLIOGRAPHIQUES}

Allcroft R., Burns K. N., 1968. Hypomagnesaemia in cattle. N. Z. Vet. J., 16, rog-I28.

Anonyme, 197x. Analytical methods for atomic absorption spectrophotometry. Perkin Elmer, Norwalk. Connecticut U. S. A.

Bois P., JASmin G., 1971. Magnésium et allergie in : 1er Symposium international sur le deficit magnésique en pathologie humaine, 1, 495-499, S. G. E. M. V. ed. Vittel.

Briscoe A. M., RAgan Ch., 1966. Diurnal variation in calcium and magnesium excretion in Man. Metabolism, 15, I002-roIo.

Darlu P., Henrotte J. G., I972. La variabilité intraindividuelle du rythme circadien du Mg urinaire. Biom. Hum., 7, I I-I9.

Doe R. P., Vennes J. A., Flink E. B., 1960. Diurnal variations of 17-Hydroxycorticosteroids, sodium, potassium, magnesium and creatinine in normal subjects and in cases of treated adrenal insufficiency and Cushing's syndrome. J. Clin. Endocr., 20, 253-265.

Drenick E. J., Hunt I. F., Swendeid M. E., ig69. Magnesium depletion during prolonged fasting of obese males. J. Clin. Endocr., 29, $134 \mathrm{I}-\mathrm{I} 348$.

Heaton F. W., Hodgkinson A., 1963. External factors affecting diurnal variation in electrolyte excretion with particular references to calcium and magnesium. Clin. Chim. Acta, 8, 246-254.

Henrotte J. G., Durlach J., I971. Magnésium et biométrie humaine. In 1er Symposium International sur le deficit magnésique en pathologie humaine, 1, $9 \mathrm{I}-\mathrm{I} о \mathrm{~g}$.

Hodgkinson A., Heaton F. W., I965. The effect of food ingestion on the urinary excretion of calcium and magnesium. Clin. Chim. Acta, 11, 354.

INGLE D. J., GRIFFITH J. Q., 1963. Surgery of the rat, in : The rat in laboratory investigation, 434-45I, I vol, 2 e ed., E. J. Farris, J. Q. Griffith, editors, Publisher Hafner Publ. Co. New York.

Kalckar H. M., I947. The enzymatic synthesis of purine riboside. J. Biol. Chem., 167, 477-482. 
Larvor P., DURLach J., I971. Rapports physiologiques entre magnésium et glandes endocrines, in $1^{\text {er }}$ Symposium International sur le déficit magnésique en pathologie humaine, 1, 25I-296. S. G. E. M. V., ed. Vittel.

Milhaud G., Perault-Staub A. M., Staub J. F., I972. Diurnal variation in plasma calcium and calcitonin in the rat. J. Physiol., 222, 559-567.

Rayssiguier Y., Larvor P., i972. Hypermagnésémie et sécrétion médullo-surrénalienne. Ann. Biol. Anim. Bioch. Biophys., 12, 479-49I.

Rayssiguier Y., Larvor P., Barlet J. P., I973. Étude de l'influence d'un phosphonate de sodium sur le taux rénal de calcium chez le Rat carencé en magnésium. C. R. Acad. Sci. (D), 276, 2035-2038.

Rizzo S. C., 1965. Variazione nictoemerali della escrezione urinaria del $\mathrm{Mg}$ in soggetti normali. Soc. Ital. Biol. Speriment, 41, 273-275.

Stutzman F. L., Amatuzio D. S., I952. A study of serum and spinal fluid calcium and magnesium in normal humans. Arch. Biochem., 39, 27 I. 